\title{
A comparison of the temperature difference according to the placement of a nasopharyngeal temperature probe
}

\author{
Hyungsun Lim, Boram Kim, Dong-Chan Kim, Sang-Kyi Lee, and \\ Seonghoon Ko \\ Department of Anesthesiology and Pain Medicine, Chonbuk National University Medical School and Hospital, \\ Jeonju, Korea
}

\begin{abstract}
Background: The purpose of this study was to compare temperatures measured at three different sites where a nasopharyngeal temperature probe is commonly placed.

Methods: Eighty elective abdominal surgical patients were enrolled. After anesthesia induction, four temperature probes were placed at the nasal cavity, upper portion of the nasopharynx, oropharynx, and the esophagus. The placement of the nasopharyngeal temperature probes was evaluated using a flexible nasendoscope, and the depth from the nares was measured. The four temperatures were simultaneously recorded at 10-minute intervals for 60 minutes.

Results: The average depths of the probes that were placed in the nasal cavity, upper nasopharynx, and the oropharynx were respectively $5.7 \pm 0.9 \mathrm{~cm}, 9.9 \pm 0.7 \mathrm{~cm}$, and $13.6 \pm 1.7 \mathrm{~cm}$ from the nares. In the baseline temperatures, the temperature differences were significantly greater in the nasal cavity $0.32(95 \% \mathrm{CI} ; 0.27-0.37)^{\circ} \mathrm{C}$ than in the nasopharynx 0.02 $(0.01-0.04)^{\circ} \mathrm{C}$, and oropharynx $0.02(-0.01 \text { to } 0.05)^{\circ} \mathrm{C}$ compared with the esophagus $(\mathrm{P}<0.001)$. These differences were maintained for 60 minutes. Twenty patients showed a $0.5^{\circ} \mathrm{C}$ or greater temperature difference between the nasal cavity and the esophagus, but no patient showed such a difference at the nasopharynx and oropharynx.

Conclusions: During general anesthesia, the temperatures measured at the upper nasopharynx and the oropharynx, but not the nasal cavity, reflected the core temperature. Therefore, the authors recommend that a probe should be placed at the nasopharynx $(\approx 10 \mathrm{~cm})$ or oropharynx $(\approx 14 \mathrm{~cm})$ with mucosal attachment for accurate core temperature measurement.
\end{abstract}

Key Words: Body temperature, Esophagus, Nasopharynx, Thermometers.

Corresponding author: Seonghoon Ko, M.D., Ph.D.

Department of Anesthesiology and Pain Medicine, Chonbuk National University Medical School and Hospital, Geonji-ro 20, Deokjin-gu, Jeonju 54907, Korea

Tel: 82-63-250-1979, Fax: 82-63-250-1240

E-mail: shko@jbnu.ac.kr

ORCID: http://orcid.org/0000-0003-2742-9985

Received: February 24, 2016.

Revised: 1st, March 21, 2016; 2nd, March 24, 2016.

Accepted: March 29, 2016.

Korean J Anesthesiol 2016 August 69(4): 357-361

http://dx.doi.org/10.4097/kjae.2016.69.4.357

\section{Introduction}

Volatile anesthetics inhibit the thermoregulatory center in the brain $[1,2]$. Because most modern volatile anesthetics have vasodilatory properties [3], blood is redistributed from the central compartment to the peripheral compartment during general anesthesia. These factors contribute to the rapid redistribution of body temperature to the peripheral compartment via blood flow. Exposure of operation fields to the low ambient temperature of the operating room can facilitate inadvertent intraoperative hypothermia. Therefore, standards of monitoring during anes-

(c) This is an open-access article distributed under the terms of the Creative Commons Attribution Non-Commercial License (http://creativecommons.org/ licenses/by-nc/4.0/), which permits unrestricted non-commercial use, distribution, and reproduction in any medium, provided the original work is properly cited. 
thesia and recovery recommend continuous core temperature monitoring during anesthesia [4], because intraoperative hypothermia can result in serious adverse effects such as myocardial ischemia, coagulopathy, and surgical wound infection [5-9].

The core temperature monitoring sites during anesthesia vary according to the purpose of measurement and the operation site. Sites in close proximity to the great vessels or highly perfused organs are the most reliable for the measurement of core temperature. Among several core temperature measurement sites, the nasopharynx, esophagus, and the pulmonary artery have been considered as the most reliable sites, reflecting accurate brain temperature [10]. However, the temperature of the central blood measured by a pulmonary artery catheter or central venous catheter is not routinely used. Because the nasopharynx is in close proximity to the internal carotid artery (ICA) and can easily be approached by anesthetists, the nasopharynx is a commonly used temperature-monitoring site during general anesthesia [11]. The placement of a nasopharyngeal temperature probe is important when to measure the core temperature accurately. However, placement in close proximity to the ICA would be difficult because the probe is placed blindly. A previous study reported that nasopharyngeal temperature probes were optimally positioned in the upper or mid-nasopharynx by residents and nurses in $43 \%$ and $41 \%$ of cases, respectively [12].

The authors hypothesized that sub-optimally placed temperature probes could not reflect the core temperature, and that actual temperatures may differ from those measured by optimally placed probes. The purpose of this study was to compare the temperatures measured at three different sites at which probes are commonly positioned.

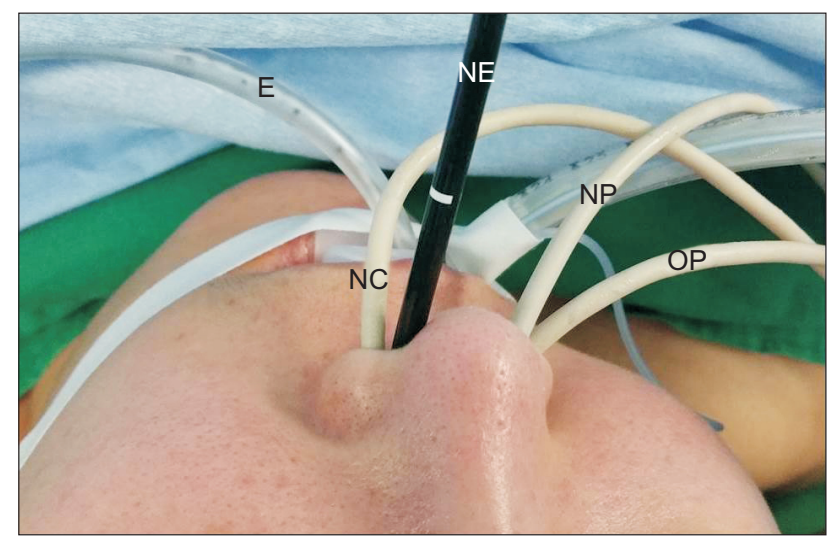

Fig. 1. Four temperature probes were placed, one each in the nasal cavity (NC), upper portion of the nasopharynx (NP), oropharynx (OP) and the esophagus (E). Probe placement was evaluated using a nasendoscope (NE).

\section{Materials and Methods}

This prospective study was approved by the Institutional Review Board of the author's institution and written informed consent was obtained from all participants. Eighty elective abdominal surgical patients, American Society of Anesthesiologists physical status 1 or 2 and aged 18-75 years, were enrolled. Patients who had a history of surgery or trauma of the nose or patients with coagulopathy, liver cirrhosis, or recurrent epistaxis were excluded.

No patients received nasal vasoconstrictor spray or preanesthetic medication. The anesthetic regimen was standardized for all patients. Patients were monitored by ECG, non-invasive blood pressure, pulse oximetry, temperature, capnography, peripheral nerve stimulator, and bispectral index (BIS). Anesthesia was induced with intravenous propofol and maintained with sevoflurane. Orotracheal intubation was performed after skeletal muscle paralysis and the cuff pressure of the tracheal tube was kept to $25-30 \mathrm{cmH}_{2} \mathrm{O}$ using a cuff pressure manometer. The ambient temperature of the operating room was maintained at 22 $\pm 1^{\circ} \mathrm{C}$ and patients were not actively warmed during the study period. Operations were performed in the supine position. After anesthesia induction, three temperature probes (M1024247, GE Healthcare, Helsinki, Finland) were placed one each at the nasal cavity, upper portion of the nasopharynx, and the oropharynx through the inferior meatus under the nasendoscopic evaluation, simultaneously. Additionally, the esophageal temperature probe (EPT1663, Ewha Biomedics, Goyang, Korea) was inserted into the lower esophagus (Fig. 1). Four temperature probes were connected to the monitor (Carescape ${ }^{\mathrm{TM}}$ Monitor B850, GE Med-

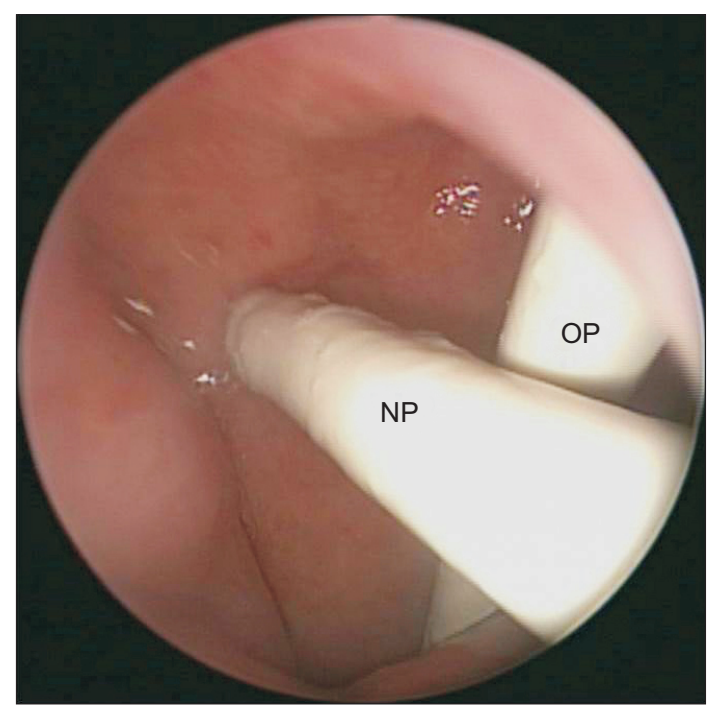

Fig. 2. The nasopharyngeal temperature probe (NP) was attached to the mucosa of the upper portion of the nasopharynx under nasendoscopic view. The oropharyngeal probe (OP) passed through the nasopharynx. 
ical Systems, Freiburg, Germany) using temperature modules (E-PT module, GE Medical Systems). All temperature probes were used after pretesting and two-point calibration at $20^{\circ} \mathrm{C}$ and $40^{\circ} \mathrm{C}$ using a digital thermometer with $0.1^{\circ} \mathrm{C}$ of tolerance (TES 1300, Taipei, Taiwan).

The investigators evaluated the placement of the temperature probes and measured the depths from the nares using a flexible nasendoscope (LF-GP, Olympus Medical Systems Corp., Tokyo, Japan). If the temperature probe was suspended in the nasopharynx, the probe was adjusted to attach to the nasopharyngeal mucosa (Fig. 2). Four temperatures including the esophageal temperature were simultaneously recorded at 10 -minute intervals for 60 minutes after anesthesia induction. The patients were warmed by a forced air warming unit (Bair Hugger Model 505, Arizant Healthcare Inc., Eden Prairie, MN, USA) after data collection and body temperatures were maintained at $36-37^{\circ} \mathrm{C}$. The data-collecting anesthesiologists did not observe the placement of the temperature probes, so that they were blind to whether the temperature was measured by the probe. Because the upper portion of the nasopharynx was in close proximity to the ICA [12], the authors concluded that the upper portion of the nasopharynx would be optimal placement for the probe. The temperature differences between the upper nasopharynx and the other two sites were evaluated, and the nasal temperatures were compared with the esophageal temperature.

\section{Statistical analysis}

The sample size was predetermined by ANOVA sample size test using SigmaPlot 12.5 (Systat Software Inc., San Jose, CA, USA) based on the assumption that the minimum detectable difference in means and expected standard deviation of the residuals were $0.1^{\circ} \mathrm{C}$ and $0.2^{\circ} \mathrm{C}$, respectively. This assumption ascertained that 77 patients were required for a significance level of $0.05(\alpha=0.05)$ and a desired power of $0.8(\beta=0.2)$. To allow for attrition, the sample size was increased to 80 .

Parametric data including demographic data and temperatures are expressed as mean $\pm \mathrm{SD}$. The temperature differences

Table 1. Depth of the Temperature Probes from the Nares $(\mathrm{cm})$

\begin{tabular}{lrc}
\hline & & P values \\
\hline Nasal cavity & & \\
$\quad$ Female & $5.3 \pm 0.7$ & $<0.001$ \\
$\quad$ Male & $6.1 \pm 1.0$ & \\
Upper nasopharynx & & \\
$\quad$ Female & $9.8 \pm 0.5$ & 0.007 \\
$\quad$ Male & $10.2 \pm 0.8$ & \\
Oropharynx & & \\
$\quad$ Female & $13.1 \pm 1.5$ & $<0.001$ \\
$\quad$ Male & $14.3 \pm 1.6$ & \\
\hline
\end{tabular}

according to probe placement are expressed as median and 95\% confidence interval (CI). Data were analyzed using oneway repeat measured analysis of variance (ANOVA) and oneway ANOVA. The Holm-Sidak method was used for multiple comparisons after ANOVA tests. A P value of less than 0.05 was considered statistically significant.

\section{Results}

Of the 80 enrolled surgical patients, of whom 45 were female and 35 were male, three patients were excluded from data analysis because two patients experienced epistaxis during the temperature probe insertion that obstructed nasendoscopic examination and one patient had a malfunctioning probe. The remaining 77 patients (male: 34 , female: 43 ) were analyzed. The average age, height, and weight were $61 \pm 15$ years, $160 \pm 10$ $\mathrm{cm}$, and $61 \pm 13 \mathrm{~kg}$, respectively. The depths of the temperature probes from the nares to the nasal cavity, upper portion of the nasopharynx, and the oropharynx are presented in Table 1.

The average time between baseline temperature measurement and endotracheal intubation was $7.5 \pm 3.4$ minutes. Operations were performed on average $13.3 \pm 6.6$ minutes after measuring the baseline temperature. The baseline temperatures of the upper nasopharynx were significantly higher than those of the nasal cavity $\left(36.4 \pm 0.4^{\circ} \mathrm{C}\right.$ vs. $\left.36.1 \pm 0.5^{\circ} \mathrm{C}, \mathrm{P}<0.001\right)$, but no difference was found compared with the oropharyngeal temperature $(\mathrm{P}=$ 0.986). In the baseline temperatures, the temperature differences compared with the esophagus were significantly greater at the nasal cavity $0.32(95 \% \mathrm{CI} ; 0.27-0.37)^{\circ} \mathrm{C}$ than at the nasopharynx $0.02(0.01-0.04)^{\circ} \mathrm{C}$, or oropharynx $0.02(-0.01 \text { to } 0.05)^{\circ} \mathrm{C}$. The

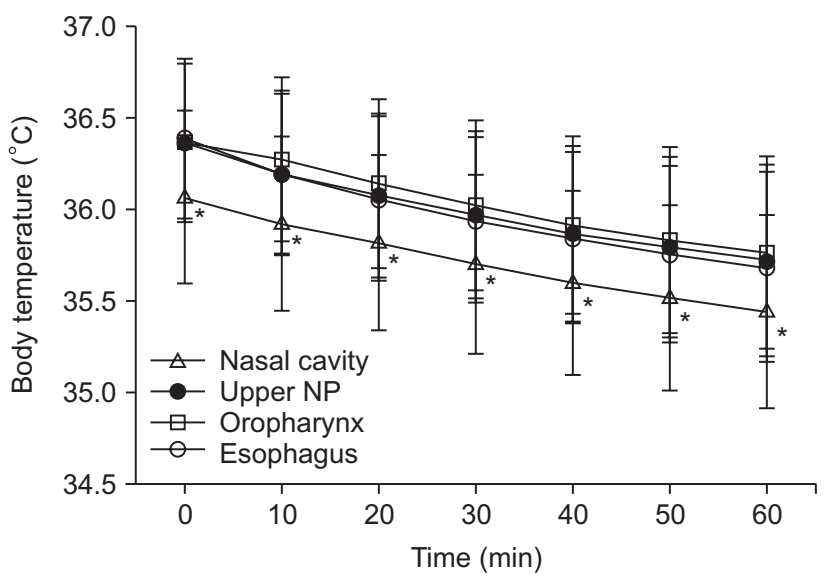

Fig. 3. Changes in body temperature were measured at four different sites. * Represents that the temperatures measured in the nasal cavity were significantly lower than at the upper nasopharynx (NP), oropharynx, and the esophagus $(\mathrm{P}<0.05)$. There were no significant temperature differences among the upper NP, oropharynx, and the esophagus. 
temperature measured at the nasal cavity was significantly lower than at the upper nasopharynx, oropharynx, and the esophagus over the study period, and these temperature differences were maintained constantly (Fig. 3). There were no significant differences between the upper nasopharyngeal temperatures and the oropharyngeal temperatures at any time point.

The number of patients who showed a temperature difference of $0.2^{\circ} \mathrm{C}$ or more compared with the esophageal temperature at baseline were 3,15 , and 58 at the upper nasopharynx, oropharynx, and the nasal cavity, respectively $(\mathrm{P}<0.001)$. Furthermore, 20 patients showed a $0.5^{\circ} \mathrm{C}$ or greater temperature difference between the nasal cavity and the esophagus, but a more than $0.5^{\circ} \mathrm{C}$ difference was not seen at the nasopharynx or the oropharynx. At 60 minutes after baseline temperature measurement, 6 and 48 patients showed $<35^{\circ} \mathrm{C}$ and $35-36^{\circ} \mathrm{C}$ esophageal temperatures, respectively.

\section{Discussion}

In the current study, the temperatures measured at the nasopharynx, oropharynx, and the esophagus were very similar, but the temperature at the nasal cavity was lower than at the other sites. Body temperatures were decreased by $0.6-0.7^{\circ} \mathrm{C}$ at the first one hour after intubation, unless the patients were not actively warmed. The appropriate position of the nasopharyngeal temperature probe is the upper portion of the nasopharynx, which is in close proximity to the internal carotid artery [12]. The depth from the nostril to the upper portion of the nasopharynx was approximately $10 \mathrm{~cm}$.

The placement of a nasopharyngeal temperature probe is important for accurate core temperature measurement. However, it is not easy for anesthetists to place the nasopharyngeal temperature probe optimally because of a lack of knowledge about optimal placement and the blind placement of the probe. In a previous study, more than half of blindly-placed nasopharyngeal temperature probes in clinical practice were found to be sub-optimal [12]. Great temperature differences were expected at the nasal cavity or oropharynx compared with the upper nasopharyngeal temperature. Although temperatures measured at the nasal cavity showed great differences when compared with the upper nasopharyngeal temperature, there were no significant differences between the oropharyngeal and upper nasopharyngeal temperatures during general anesthesia unlike the authors' hypothesis. Therefore, superficial insertion of the probe placed in the nasal cavity is less accurate than deep insertion of the probe placed at the oropharynx if the probe is not placed optimally at the upper nasopharynx. It might be caused by the increased blood flow in the oropharyngeal mucosa caused by the vasodilatory effect of volatile anesthetic during general anesthesia, although there is no great vessel near the oropharynx.
However, the temperature measured at the nasal cavity was lower than the temperature measured at the other sites. The lower temperature at the nasal cavity may be caused by the effects of ambient temperature, because the inserted depth of the probe was more superficial than at the other sites, and some probes were not attached to the nasal mucosa and thus were suspended in the nasal cavity.

In a volunteer study, the core temperature decreased $1.6^{\circ} \mathrm{C}$ in the first hour of anesthesia due to the internal redistribution of heat [13]. In the current result, the esophageal and nasopharyngeal temperatures declined by $0.6-0.7^{\circ} \mathrm{C}$ for 1 hour. The ambient temperatures were similar $\left(\approx 22^{\circ} \mathrm{C}\right)$ and the subjects were not warmed actively in either study. The patients had cloth draped over the chest and extremities in the current study, but the volunteers were minimally clothed and fully exposed to the operating room temperature in the previous study. The extremities are important peripheral thermal compartment that can induce core hypothermia via heat redistribution during the first hour after anesthesia induction [13,14]. The great decrease in core temperature in previous study could be direct results of full exposure to ambient temperature without an operation drape.

Although a nasopharyngeal temperature probe can be inserted through the inferior or middle meatus in the nasal cavity, probes were inserted through the inferior meatus in the current study. The average depths of the optimally placed probe from the nares were $9.8 \mathrm{~cm}$ in females and $10.2 \mathrm{~cm}$ in males. In the previous study, a $9.5-10.0 \mathrm{~cm}$ probe depth was recommended for optimal placement, as in the present results [12]. The temperature probe should be attached to the nasopharyngeal mucosa to reflect the blood temperature. The probe can be influenced by the ambient temperature if the probe is suspended in the nasal cavity. Therefore, appropriate depth and mucosal contact are important for the optimal placement of the nasopharyngeal temperature probe. The authors speculated that some temperature probes in the nasal cavity were suspended and more affected by the ambient temperature than the nasopharyngeal or oropharyngeal probes. However, nasopharyngeal and oropharyngeal probes can be suspended although the possibility was lower than the nasal cavity in the anatomical aspect. If the probe was not attached to the mucosa at the nasopharynx or oropharynx, the measured temperature may be lower than the temperature as measured by a probe with mucosal contact. The esophageal temperature was measured as the other standard of core temperature because an esophageal temperature probe can maintain more stable mucosal contact than a nasopharyngeal probe.

There are several limitations in the current study. First, patients were not actively warmed during the study period. Perioperative hypothermia is associated with adverse clinical effects. Although six patients' esophageal temperatures decreased to lower than $35^{\circ} \mathrm{C}$ at 60 minutes after temperature measurement, 
all patients' body temperatures were kept at $36-37^{\circ} \mathrm{C}$ by a forced air warming unit after the study period. Second, the mucosal contact of the probes in the nasal cavity and oropharynx was not evaluated. If the temperatures were compared according to mucosal contact, the cause of the temperature difference could be clearer. Third, subjects were limited to patients who underwent open abdominal surgeries. In general, loss of body heat is greater in open abdominal surgery due to the larger amount of evaporation from abdominal contents compared to extremity surgery. Consequently, it is difficult to extrapolate the present results to all types of surgery, because of physiological and surgical differences.

In conclusion, the temperatures measured at the upper nasopharynx and the oropharynx reflect the core temperature during general anesthesia, but the temperature from the nasal cavity does not. Therefore, the authors recommend that the probe should be placed at the nasopharynx $(\approx 10 \mathrm{~cm}$ depth from the nares) or at the oropharynx $(\approx 14 \mathrm{~cm}$ depth) with mucosal attachment for accurate nasopharyngeal temperature measurement.

\section{References}

1. Støen R, Sessler DI. The thermoregulatory threshold is inversely proportional to isoflurane concentration. Anesthesiology 1990; 72: 822-7.

2. Atarashi K, Ozaki M, Suzuki H. Sevoflurane comparably decreases the threshold for thermoregulatory vasoconstriction as isoflurane. Masui 1996; 45: 818-23.

3. Akata T, Nakashima M, Izumi K. Comparison of volatile anesthetic actions on intracellular calcium stores of vascular smooth muscle: investigation in isolated systemic resistance arteries. Anesthesiology 2001; 94: 840-50.

4. Birks RJ, Gemmell LW, O'Sullivan EP, Rowbotham DJ, Sneyd JR. Recommendations for standards of monitoring during anaesthesia and recovery. 4th ed. London, The Association of Anaesthetists of Great Britain and Ireland. 2007.

5. Kurz A, Sessler DI, Lenhardt R. Perioperative normothermia to reduce the incidence of surgical-wound infection and shorten hospitalization. Study of Wound Infection and Temperature Group. N Engl J Med 1996; 334: 1209-15.

6. Reed RL 2nd, Johnson TD, Hudson JD, Fischer RP. The disparity between hypothermic coagulopathy and clotting studies. J Trauma 1992; 33: 465-70.

7. Frank SM, Fleisher LA, Breslow MJ, Higgins MS, Olson KF, Kelly S, et al. Perioperative maintenance of normothermia reduces the incidence of morbid cardiac events. A randomized clinical trial. JAMA 1997; 277: 1127-34.

8. Frank SM, Beattie C, Christopherson R, Norris EJ, Perler BA, Williams GM, et al. Unintentional hypothermia is associated with postoperative myocardial ischemia. The Perioperative Ischemia Randomized Anesthesia Trial Study Group. Anesthesiology 1993; 78: 46876.

9. Schmied H, Kurz A, Sessler DI, Kozek S, Reiter A. Mild hypothermia increases blood loss and transfusion requirements during total hip arthroplasty. Lancet 1996; 347: 289-92.

10. Stone JG, Young WL, Smith CR, Solomon RA, Wald A, Ostapkovich N, et al. Do standard monitoring sites reflect true brain temperature when profound hypothermia is rapidly induced and reversed? Anesthesiology 1995; 82: 344-51.

11. Torossian A. Survey on intraoperative temperature management in Europe. Eur J Anaesthesiol 2007; 24: 668-75.

12. Lee J, Lim H, Son KG, Ko S. Optimal nasopharyngeal temperature probe placement. Anesth Analg 2014; 119: 875-9.

13. Matsukawa T, Sessler DI, Sessler AM, Schroeder M, Ozaki M, Kurz A, et al. Heat flow and distribution during induction of general anesthesia. Anesthesiology 1995; 82: 662-73.

14. Sessler DI. Mild perioperative hypothermia. N Engl J Med 1997; 336: 1730-7. 